\title{
Advances and applications for automated drones in underground mining operations
}

\author{
E. Jones ${ }^{1}$, J. Sofonia' ${ }^{1}$, C. Canales ${ }^{2}$, S. Hrabar ${ }^{1}$, and F. Kendoul' ${ }^{1}$ \\ ${ }^{1}$ Emesent, Brisbane, Australia \\ 2Universidad de Concepción, Chile
}

\begin{abstract}
The development and current state of the Hovermap autonomous flight system in underground and GPS-denied areas is discussed, with examples obtained during the development and early adoption of the system. The current performance of the system and subsequent data interpretation suggest some scenarios in which Hovermap deployment is appropriate and have been proven. The examples focus principally on improving safety through a better understanding of the rock mass behaviour and failure mechanisms commonly encountered in deep and high-stress mining conditions, and from feeding these insights back into the design process. Recent and future developments in the hardware and software platforms and the associated data analytics are also outlined
\end{abstract}

\section{INTRODUCTION}

Mining within deep and high-stress conditions inherently involves risks both to the personnel and the mining operation. To operate safely and efficiently in such extreme environments, it is necessary to understand the behaviour of the rock mass in response to the continuously changing stress field. Mobile scanning data acquisition methods and interpretations have emerged since 2012 and have been shown to improve the decision-making processes of mining and geotechnical engineers, improving both safety and design. More recently, data acquisition has utilised autonomous and semi-autonomous drones. Just as mobile scanning provided new data for human-inaccessible areas, drones are now advancing this methodology to previously inaccessible areas.

\section{OVERVIEW OF THE DEVELOPMENT OF HOVERMAP}

Underground mining presents many accessibility challenges. As mines become deeper, hazards such as seismicity increase. The appetite for companies to allow exposure to such hazards is decreasing, yet an increasing focus on safety mandates more inspections. The development of the Hovermap mobile mapping and drone autonomy platform breaks this impasse by allowing access and inspection without compromising the safety of personnel. The solution is a payload that allows commercially available drones to fly in GPS-denied environments.

For many years, the CSIRO has been at the forefront of research into industrial robotics, as well as the development of 3D simultaneous localization and mapping (SLAM) algorithms, Zlot and Bosse (2012). A specialised group was formed to combine these two specialties to enable autonomous drone flights in GPS-denied environments. 
Emesent, a spin-out of CSIRO, has commercialised Hovermap by utilising real-time 3D SLAM algorithms and advanced drone autonomy technologies to provide drone automation while generating a high-resolution point cloud of its flight path environment.

\section{DESIGN AND ENGINEERING OF AN AUTONOMOUS DRONE SYSTEM}

Hovermap is a detachable payload mounted beneath a drone. It comprises of a multi-channel LiDAR, inertial measurement unit (IMU), and an onboard computer. The LiDAR sensor is mechanically rotated, changing the intended output from a $360^{\circ}$ planer field of view, into a $360^{\circ} \times 360^{\circ}$ spherical field of view. For position recognition and sensing, the LiDAR is coupled with a low-grade micro-electricalmechanical system (MEMS) IMU. The IMU data and LiDAR results are processed on-board in real-time within the SLAM algorithm to generate the point cloud of the scanned environment. This SLAM solution can interface with compatible drones, providing omni-directional collision avoidance, position hold and pre-programmed flights in GPS-denied and dark environments. Table I summarises the parameters of Hovermap.

Table I. Summary of the Hovermap parameters

\begin{tabular}{|c|c|c|}
\hline & Description & Comment \\
\hline \multicolumn{3}{|c|}{ Sensor } \\
\hline \multirow[t]{5}{*}{ LiDAR } & Velodyne VLP-16 Lite (Puck) & \multirow{5}{*}{$\begin{array}{l}\text { LiDAR rotates to produce } 360^{\circ} \times 360^{\circ} \text { field of view. } \\
\text { Operation range is typically } 60-80 \mathrm{~m} \text {. } \\
\text { Points emitted. Returns depend on environment. } \\
\text { Useful for complex environments. }\end{array}$} \\
\hline & Up to $100 \mathrm{~m}$ range & \\
\hline & 16 Channels & \\
\hline & - $\quad 300,000$ points/sec & \\
\hline & Dual return & \\
\hline \multicolumn{3}{|c|}{ Drone } \\
\hline Type & $\begin{array}{lrrr}\text { DJI Wind 2, } & \text { Wind } 4, \\
\text { M200/210, M600. } & \end{array}$ & Depends on flight type and required duration. \\
\hline Flight time & $10-30 \mathrm{~min}$ & Dependent on drone and flying environment. \\
\hline IP rating & Variable: none - IP57 & Drone dependent. \\
\hline \multicolumn{3}{|c|}{ Mapping and Results } \\
\hline SLAM & $\begin{array}{l}\text { Simultaneous localisation and } \\
\text { mapping algorithm }\end{array}$ & $\begin{array}{l}\text { Used for collision avoidance, flight path mapping, } \\
\text { point cloud results generation. }\end{array}$ \\
\hline $\begin{array}{l}\text { Point cloud } \\
\text { processing time }\end{array}$ & $\begin{array}{l}\text { Local: Real time during flight } \\
\text { Global: Less than duration of } \\
\text { flight }\end{array}$ & $\begin{array}{l}\text { Collision avoidance and flight path. } \\
\text { Full point cloud generation for post-flight } \\
\text { analytics. }\end{array}$ \\
\hline $\begin{array}{lr}\text { Point } & \text { cloud } \\
\text { Output } & \text { and } \\
\text { attributes }\end{array}$ & .laz and .ply & $\begin{array}{l}x, y, z \text {, intensity, range and channel attributes for } \\
\text { each return, plus flight path pose. }\end{array}$ \\
\hline Accuracy* & $\pm 30 \mathrm{~mm} / \pm 15 \mathrm{~mm}$ & $\begin{array}{l}\text { Raw data (limited by sensor output at a distance of } \\
100 \mathrm{~m} \text { ) / following postprocessing methods and } \\
\text { limited range in underground environments. }\end{array}$ \\
\hline Precision* ${ }^{*}$ & $\sim \pm 10 \mathrm{~mm}$ & Repeatability for local point cloud alignment. \\
\hline Georeferencing & Various methods & $\begin{array}{l}\text { Spherical survey markers, alignment to existing } \\
\text { survey, visual (such as paint marks and writing). }\end{array}$ \\
\hline
\end{tabular}

* For a detailed discussion on these topics for underground mobile mapping refer to Jones et al (2018)

Two LiDAR orientations, vertically (facing downwards) and horizontally (facing forwards) have been developed and tested for underground use. Initial trials utilised the vertical orientation setup (See Figure 1). A review of the results showed that this orientation lead to a low point density in areas vertically above the drone such as the development backs, stope crowns and draw point hang-ups. A forward-facing, horizontal orientation was developed, relocating the shadow from above the drone to 
behind the drone in areas previously scanned. This orientation has proven more favourable in mining applications.
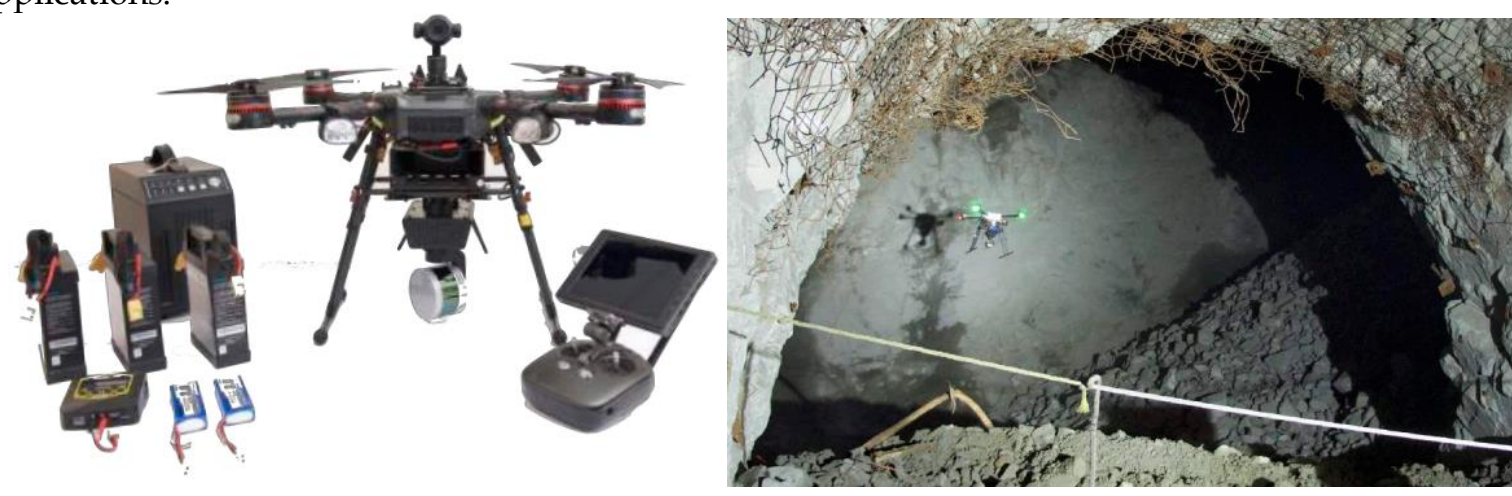

Figure 1. A) - The Hovermap used throughout the trials. B) Flying through an open draw point into a large stope

\section{METHODS FOR ATTAINING RESULTS}

The acquisition of results for analysis with Hovermap can be summarised by four steps, as outlined in Figure 2.

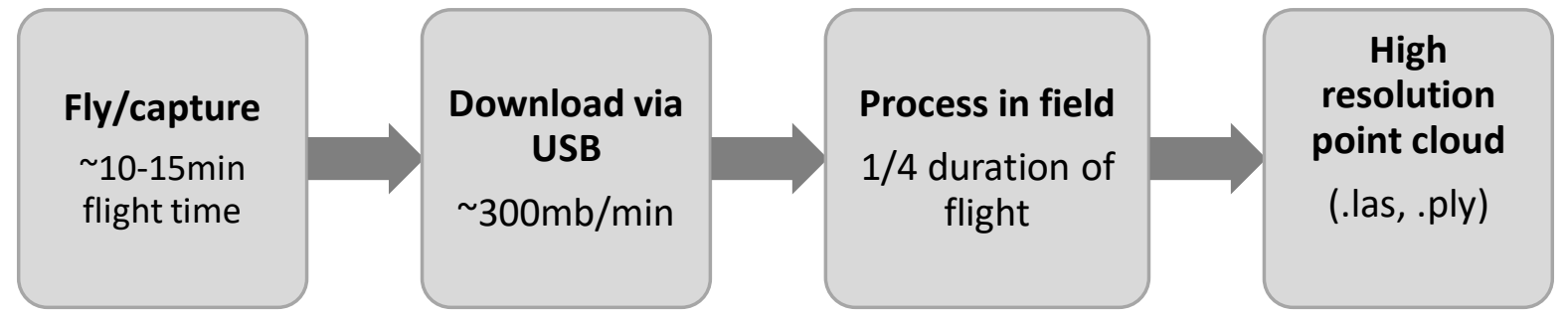

Figure 2. An overview of the steps required for attaining data from Hovermap

Hovermap provides various levels of automation to a drone. Within this study three methods of flight automation were demonstrated in mining environments:

- Autonomy level 1 - Pilot assisted mode

- Omnidirectional collision avoidance (Virtual bubble surrounding the drone)

- GPS-denied position hold and flight

- Enables safe flight close to structures in GPS-denied environments

- Autonomy level 2a - Waypoint mode

- Set waypoints in an existing map for routine pre-programmed GPS-denied flight

- Enables beyond line of sight flight with almost no input from pilot

- Autonomy level $\mathbf{2 b}$ - Tap-to-fly interactive waypoint mode in live stream map

- Set waypoints in a map streamed directly from Hovermap during a flight

- Includes collision avoidance

- Enables beyond line of sight flight with minimal input form pilot

The duration of the flight is dictated either by the mission required, the area of data capture, or by battery life. Typical underground flight speeds are 1 to $2 \mathrm{~m} / \mathrm{sec}$. For applications such as flying within a stope, the required flight duration is approximately 5 minutes, depending on the size of the stope, distance to stope access and the required point density within the stope.

Following a flight, data is transferred from the onboard computer to a secondary processing computer. Onboard storage of $480 \mathrm{~Gb}$ allows for a full day of flying, data acquisition being at approximately 
$300 \mathrm{Mb} / \mathrm{min}$. A full day of scanning therefore requires only periodic battery changes.

The processing of the SLAM is conducted in two stages. The in-flight local solution processed on-board assists with collision-avoidance and the real-time placement of waypoints to aid navigation in the GPSdenied environment. The second stage of SLAM processing is conducted after the flight providing a global SLAM solution. If multiple flights have a region of overlap, the processing software merges the scans.

The final output from the post-processing is a high-resolution point cloud containing all of the returns received from the unit, as well as the trajectory of the sensor. The data format is non-proprietary (open source format) as a .las/.laz and a .ply file. Each data set contains multiple advanced attributes as outlined in Table I.

Each attribute can be used within a data filter, thus ensuring that only the highest quality data is used for subsequent analytics. As the data is in standard point-cloud format, subsequent analysis can be conducted in many commercially available or open-source software packages.

\section{GEOREFERENCING POINT CLOUD RESULTS}

Many mining applications can benefit greatly from the registration of the point cloud into the mine coordinates. This allows scans to be interpreted with additional information such a lithology, structure, void, or the output from numerical modelling of parameters such as stress. Mapped excavations and stopes can be used for the generation of as-built shapes for survey purposes. Multiple methods of georeferencing exist, the method chosen depending on application and site-specific requirements, and the available information. A combination of methods can also assist in the efficient alignment of data. Some applications, however, may not require the georeferencing of scans. The use of survey spheres and the iterative closest point (ICP) approach, is discussed.

\section{Survey spheres}

Survey spheres have been used successfully for the purpose of georeferencing a point cloud to a mine's coordinates, but the approach is more time-consuming than ICP methods. The method involves placing three or more survey prisms attached to the wall (See Figure 3). The coordinates of the collar or the centroid of the sphere are surveyed using traditional methods. During the mobile scan, a high density of points are scanned around each sphere, providing an easily recognisable feature within the point cloud. During post-processing, a sphere of the same size as the target is fitted to the high density of points and the centroid aligned to the corresponding surveyed location (See Figure 4).

The accuracy of the scan alignment depends on both the accuracy of the alignment of the fitted sphere to the point cloud, as well as the location of spheres within the scan. Placing four or more spheres throughout the scan constrains the degrees of freedom of the alignment and improves the accuracy of the final point cloud registration. It has been found that highly reflective spheres reduce the number of LiDAR returns as it saturates the sensor, compromising number and accuracy of the returns.

The use of survey spheres is a manual process of alignment. It has the advantage that the centroid or collar of the sphere is at a known location within the mine coordinate system. These known locations allow for the calculation of the global accuracy of the final alignment of the scan. The disadvantage is that additional time is required to place the markers in the field, as well as extra time to conduct the alignment after post-processing. 

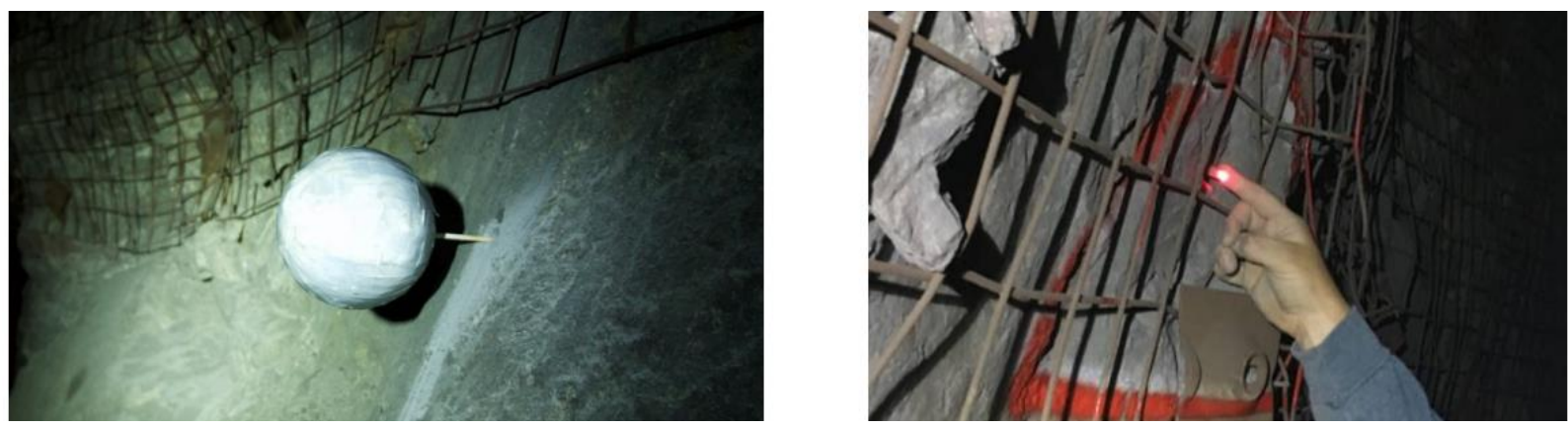

Figure 3. (a)A survey sphere is placed on the wall. (b)Either the collar or the sphere centroid location is known so the point cloud can be referenced to these locations

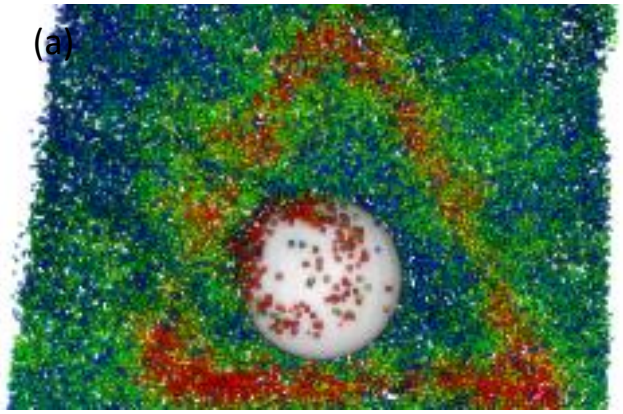

Figure 4. (a) - The results from a Hovermap point cloud and the alignment of a survey sphere. (b) Highly reflective spheres can produce noisy data reducing the accuracy of the fitted sphere

\section{Iterative closest point registration (Point cloud to point cloud or point cloud to mesh)}

Iterative closest point (ICP) registration, is an iterative method for aligning a point cloud to either a known solid shape, such as a 3D mesh, or to another point cloud. The algorithm is essentially a 3D residual mean square method in the sense that a predetermined allowable error is set and the algorithm will continue to reduce the residual error until either the threshold is met, or a maximum number of iterations have been conducted. This method works very well within an underground mining environment as each excavation has a number of features and adjoining intersections. These intersections constrain the final alignment of point cloud registration improving the accuracy of the final alignment to mine coordinates.

This method works particularly well with Hovermap data as the LiDAR sensor and SLAM algorithm used have proven to produce point clouds with far fewer drift errors as described by Jones et al (2018). This means the use of ICP alignment methods can align Hovermap results into mine grid very rapidly when another known shape is available.

\section{Summary of georeferencing}

While there are several methods available for georeferencing which all require a manual step, fullyautomated methods are currently being developed. The best method depends both on the available information and on the required accuracy of the final alignment. For many standalone analytical solutions, such as stope volumes or convergence measurements, there is no absolute requirement for registration. These analytics may however also offer insights into the interpretation of larger scale mechanisms such as the influence of lithology or discontinuities. Even then, highly accurate registration is not necessarily imperative, as the interpretation of lithological boundaries and structure may contain greater uncertainty than the registration of the scan. For applications, such as the location of an advancing face for survey purposes, the accuracy of the registration is more relevant. The appropriate approach is often dictated by the required global accuracy. 
The local and global accuracies and precisions of mobile laser scanning results is a much-discussed topic. Given the larger number of variables in producing the results and variations between devices it is difficult to define, and even more difficult to quantify. It is recommended that prior to choosing a device for a particular application, it is trialled in the specific mine to confirm its appropriateness. For a fuller discussion see Jones et al (2018).

\section{CASE STUDIES FOR IMPROVING MINING SAFETY, PRODUCTIVITY AND EFFICIENCY}

Increasing workplace safety is a global requirement in underground mines. Elimination of people from hazardous environments is the top order within the hierarchy of controls and is one of the benefits of autonomous systems. In many applications where mobile scanning methods are applicable, advantages are gained by excluding people from hazardous locations, and allowing greater coverage for improved and more in-depth analytics for decisions making. Selected underground case studies are presented below, providing an overview of applications for improving safety and related insights.

\section{Stope analysis}

Initial applications of Hovermap have focused on stope inspections and analysis, the benefits arising from a safety and efficiency perspective during data capture and an analytics and operational perspective from the results set.

During the data capture of a stope, safety is improved through a reduction in exposure in both proximity and duration to the surveyors undertaking the inspection. Current cavity monitoring systems (CMS) require surveyors to access the stope as close as possible to the drawpoint or edge of the stope so the CMS scanner can be positioned as far into the stope as possible. At the drawpoints this can expose the surveyors to sudden rock fall hazards within the stope. Access to upper levels of an open stope can expose them to any unknown undercutting if it has occurred. At the completion of a CMS scan, the surveyor must then retrieve the device, exposing them for a second time. A major advantage from an exposure perspective is that the drone can be operated from a safe distance from the stope, completely removing the exposure required by current CMS methods. The efficiency of data capture is improved as the duration to setup, fly into the stope and return is typically less than 10 minutes. The result from a 5-minute flight within a stope, produces an evenly distributed, high resolution point cloud for detailed analytics.

Improvements in data analytics occurs as the drone is able to fly beyond visual line of sight (BVLOS) past the brow into the stope or hung-up drawpoint. This unprecedented access provides a detailed view of brow conditions and other occluded areas within the stope, thereby significantly reducing shadowing within the final results. In comparison, current CMS methods use a rotating laser positioned at a static location within a stope. The distribution of measurements results in a high density of data close to the scanner and diminishing further away from the scanner. The spatial resolution of CMS data varies from thousandth's to singular points per square meter. In comparison, Hovermap data is consistently in the range of ten-twenty thousand points per $\mathrm{m}^{2}$ throughout the entire stope. For a comparison between current CMS and Hovermap results for the same stope, see Figure 5.

Further analytics can be conducted on the high-resolution point cloud. The stope volume can be reconciled with greater confidence for an indication of production tonnes and required backfill (where applicable). The analysis of over- and under-break can be calculated for each individual measurement providing greater insights into the volume of both, and also the mechanism by which it has occurred.

The precision and density of the Hovermap point clouds allow for the recognition of geological structures (See Figure 6). Structural traces and planes within a stope provide geotechnical engineers with insight into the mechanisms responsible for over and under-break. This level of detail for analysis has previously been rare, given access limitation. Figure 7 shows the data collected by Hovermap and 
the recognition of geological structures allowing the determination of principal features such as joint sets, dip/dip direction, persistence, roughness and spacing. In this example, over 80 individual discontinuities were identified which can be used for further analysis by engineers and geologists.

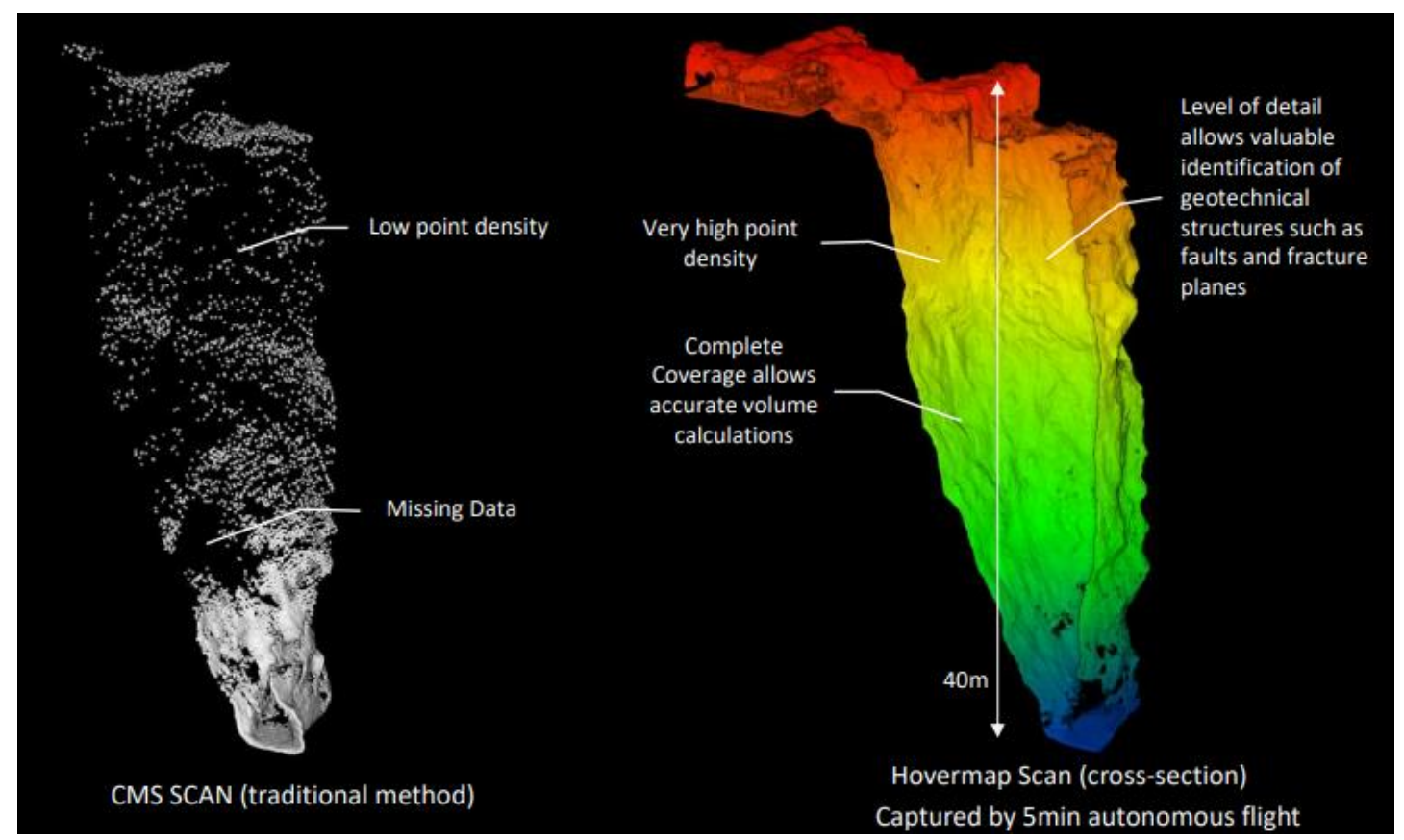

Figure 5. Comparison of the level of detail and accuracy of the final stope shape between traditional CMS and Hovermap scans
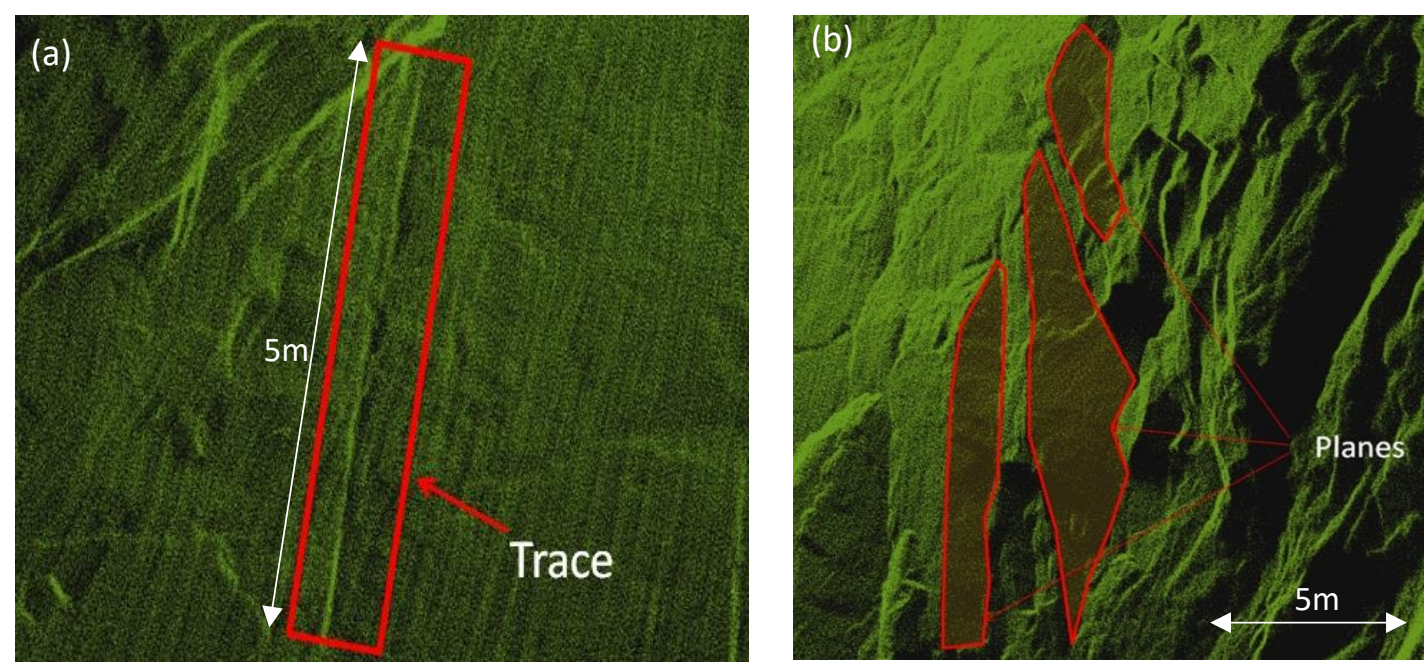

Figure 6. The point cloud density from Hovermap provides sufficient detail for the recognition of (a) structure traces and (b) planes

Larger scale structures, such as those identified in Figure 8, can provide geologists with greater insight and confidence in defining the structural model for the mine. The recognition and prediction of localised structures through a detailed structural assessment, aids the rock mass classifications for excavation stability. For geotechnical engineers, this can assist in excavation support designs and stope dimensions. Stope dimensions and blast patterns can be adjusted to accommodate the resulting increased knowledge of the rock mass characteristics. 
The rapid acquisition of stope data greatly assists the trial sites with improved scheduling and control on stopes. Sites are able to more regularly monitor stope volumes by providing greater insights into the remaining tonnes and reduced feedback in the event a stope is chimneying or caving.
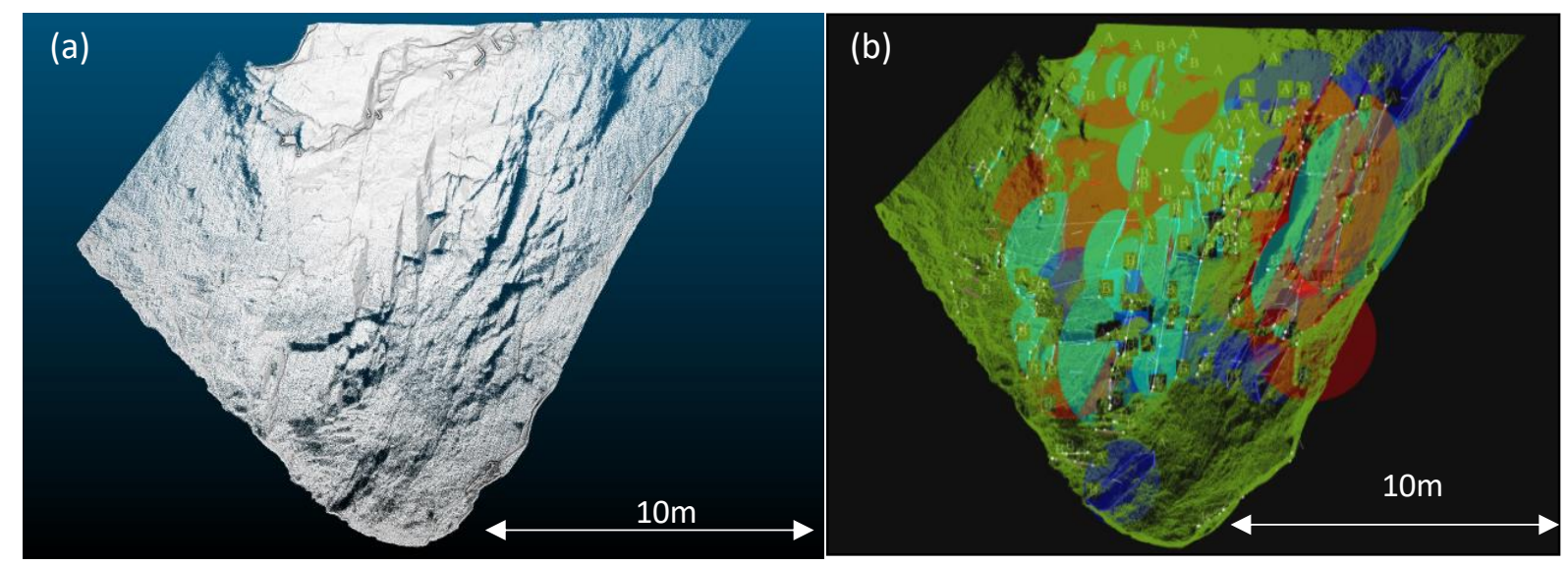

Figure 7. (a) - A stope hangingwall showing the resolution of data achieved with Hovermap. (b) The planar facets recognised from commercially available software (Sirovision)

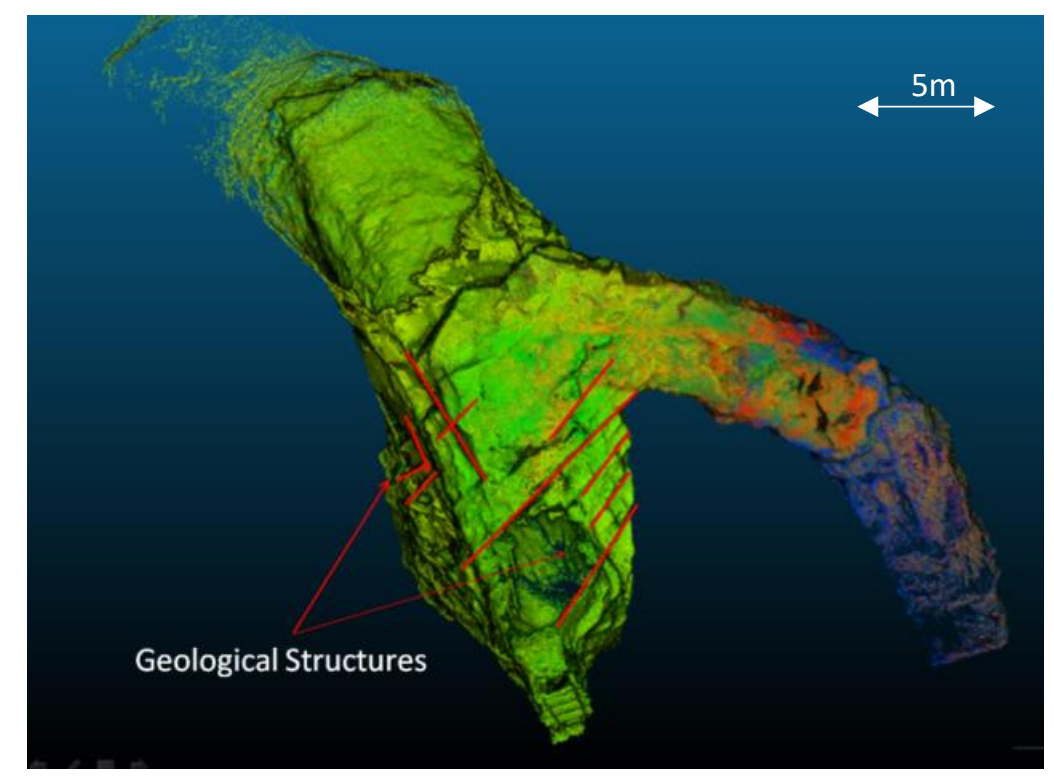

Figure 8. Large scale geological structures identified from Hovermap data within a stope

\section{Backfill monitoring}

Backfilling of stopes is used for a variety of purposes, and is largely attributed to the requirement for achieving regional ground control within a mining area. The mode of support in any of the three instances discussed by Brady and Brown (2004, p. 408, ND), is related to a combination of the rock mass characteristics, their mode of deformation, and the engineered material properties of the fill. In many high-stress mining environments where a high extraction ratio is planned, backfill is used a support element to control unravelling and consequential dilution and also assist in global stability by acting similarly to a pillar controlling hangingwall and footwall closure. In cases where backfill is being used for engineering purposes, the monitoring of the material in high detail can be used to ensure the correct design is being followed. Where a higher strength plug is formed, the accurate measurement of the fill can be used to confirm the correct placement of the material in all areas of the stope. This same confirmation of design can be applied to sites where development is planned back through a backfilled 
stope, reducing the risk of breaking through to a lower strength material. An example of a stope in the process of backfilling is shown in Figure 9.

In cases where the backfill is not used as an engineered material, regular inspections of backfill height can show the remaining volume available for waste. This knowledge can directly feed into the scheduling requirements for the mine.

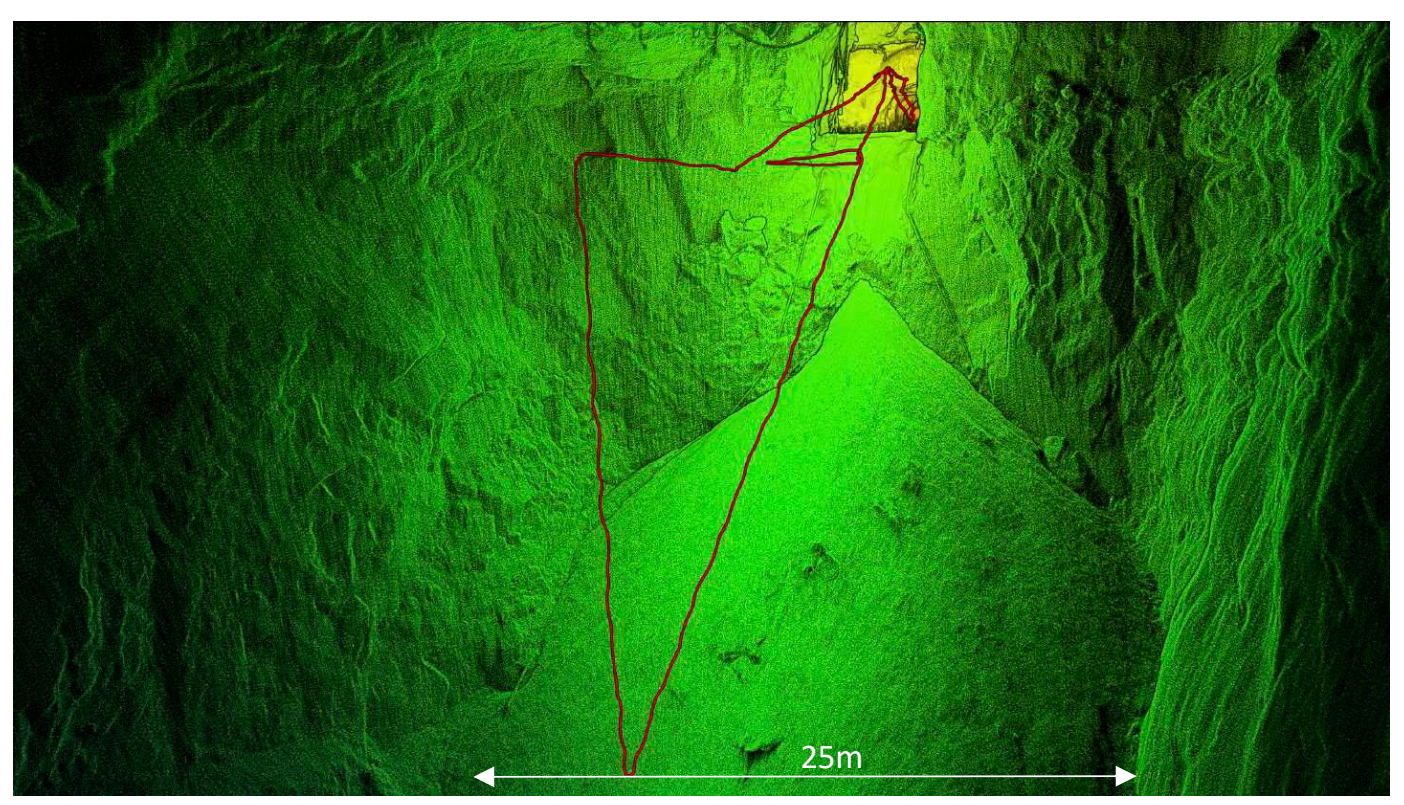

Figure 9. A dataset showing backfill height

\section{Hang-up inspections}

Drawpoint hang-ups in both stoping mines and caving mines pose a safety hazard to those attempting to clear them and come at a great cost in lost production. Current methods for clearing include blasting and hosing in an attempt to generate movement around the blockage, eventually dislodging the material blocking the drawpoint. Using Hovermap results, mining professionals can gain a clear view of any blockage, allowing a targeted approach to removing them. The example shown in Figure 10 is from a block cave where the drone pilot was located in the extraction drive, away from the drawpoint and safe from any potential movement. In this example, the Hovermap payload was in a vertical facing alignment, meaning that areas directly above the drone were occluded. This highlights the importance of selecting the correct orientation of the sensor for the required task. A horizontal, forward-facing orientation would have achieved a higher point density in the backs, creating a clearer image of the hang-up. 


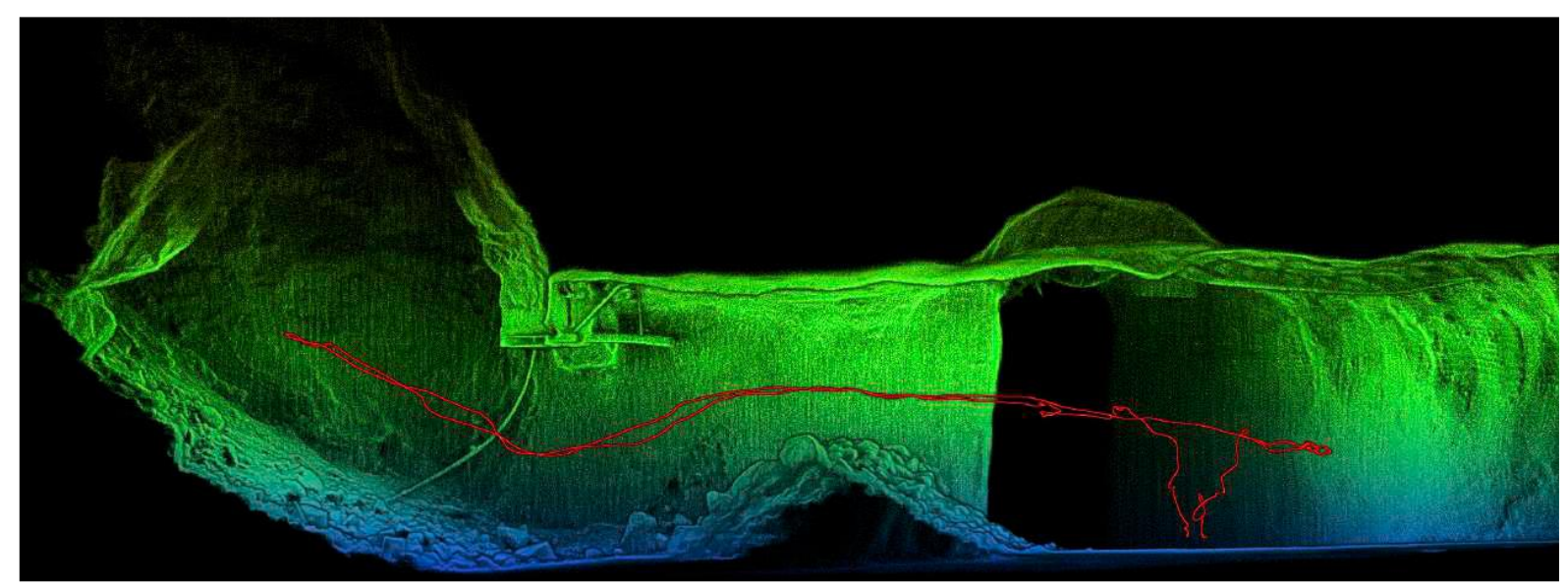

Figure 10. An example of a scan showing a drawpoint hang-up with the red line indicating the drone's flight path

Deformation monitoring and ground support analysis

The majority of personnel risk exposure is throughout development drives within a mine. Mining professionals place great importance on the ground support for providing a safe working environment, particularly in deep and high stress mines that are typically subject to dynamic deformation. Jones et al (2017), describe mobile scanning hardware and methods for deformation monitoring within underground mines. The accuracy of mobile scanning devices, such as Hovermap, are sufficient for the recognition of convergence trends and indicating areas of changes exceeding approximately $10 \mathrm{~mm}$. For some stiff rock masses that exhibit rapid/brittle failure, these trends may not be observed using this method. However, in most cases where mobile scanning has been implemented, the change detection from mobile scanning methods have provided greater insights than broad scale observational mapping greater coverage than the sub-millimetre extensometer monitoring the sites had conducted.

The results from mobile LiDAR provide a range of benefits for deformation monitoring. The trends created from multiple scans indicate closure rates which in-turn provide a guidance for the residual capacity and scheduling for rehabilitation. The displacements provide quantitative data for administrative controls such as trigger action response plans (TARPs). With the inclusion of intensity as a point cloud parameter, the distinction between rock and metal is evident (See Figure 11), allowing for improved methods of QA/QC and insights into whether the ground support is acting as a system or as individual elements. New and exciting analytical methods are being developed to better understand rock mass deformation now that near-spatially continuous, accurate data can be collected mine-wide using mobile scanning systems.

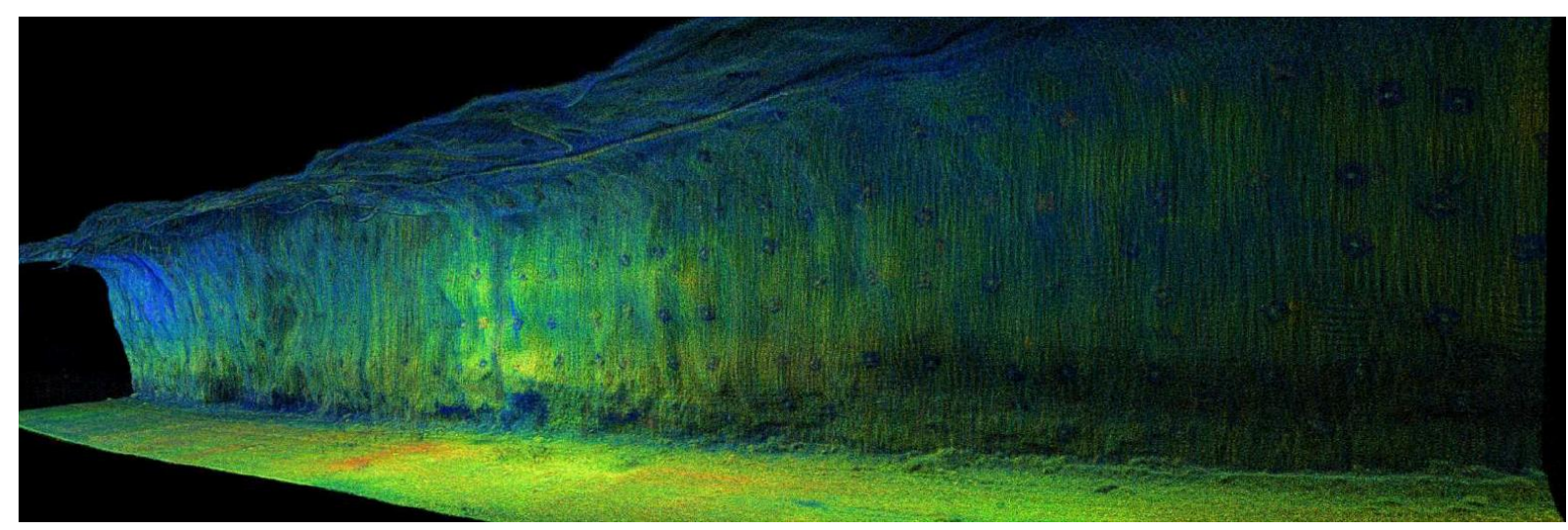

Figure 11. An example of clarity of results collected from a single scan of a drive. Colour denotes the intensity of the returning waveform highlighting the location of metal bolt plates 


\section{Summary of case studies}

The applications of the data collected by Hovermap are extensive, improving safety, efficiency and productivity in underground mines. The case studies outlined above demonstrate just some of the many ways the data may be used by mining and geotechnical engineers, surveyors and geologists. The enhanced datasets obtained from the use of Hovermap in underground operations is leading to advanced ongoing research, allowing for the continuous advancement of mining applications.

\section{THE FUTURE OF AUTOMATED DRONES IN UNDERGROUND MINING}

The use of autonomous drones in underground mining is in its infancy. However, the research to further improve their applications continues. This research, that will soon be deployed, includes:

- Autonomy level 3 - Autonomous exploration

- Single-click mission execution.

- No waypoints, just point - click - explore - return.

- SLAM-based auto registration

- SLAM-based auto registration utilises the metadata collected by hardware to join multiple scans and create a single result, as though it were a single scan.

- Quick turnaround on results requiring change detection.

- Colourisation of point clouds

- Provides additional data for classification.

- Photographic like imagery for interpretation.

- Additional sensors

- Gas detection.

- Thermal imaging cameras.

- Hyperspectral cameras.

- Global positioning system (GPS) / Real-time kinematic (RTK) positioning.

- Remote deployment

- Search and rescue missions.

- Remote re-entry following blasting or seismic events.

The analytics aspect to these hardware improvements will result in greater insights into all aspects of the mining operation. However, this requires the development of both IT infrastructure and software capable of storing, analysing and visualising the detailed datasets.

The current applications of drones and mobile sensor technology is in its infancy within underground mining. Their incorporation into underground work environments will improve safety, efficiency and productivity while improving our knowledge base in all disciplines of mining.

\section{DISCUSSION AND CONCLUSION}

The adoption of mobile mapping techniques have been increasing since the commercial release of the Zeb-1 device in 2014. The results from this method of data acquisition provides valuable insights for mining engineering designs. Systems previously available were either required to be operated by hand or were vehicle mounted. However, these methods did not allow for any additional information to be acquired in access-restricted environments.

Hovermap has been developed as a drone-mounted payload, providing collision avoidance and drone autonomy, whilst the resulting 3D point cloud can be georeferenced for analytics. This system addresses 
the issue of remote access into areas that previously were inaccessible, or deemed too hazardous for mining personnel to access. The combination of autonomy, advanced data acquisition and highresolution 3D mobile mapping is a unique combination allowing for new insights into underground mine engineering, and improved safety and mine design.

The site trials throughout the development phase, and commercial deployment since, have produced results that have directly improved safety, efficiency and productivity in the adopting mines. The examples provided above, are some of the more obvious examples of the applications of the data collected. More advanced analytics using these insights have also been implemented since these primary trials. Research and development into additional applications and the automation of processes is ongoing and a major part of the future development of Hovermap and the data it produces.

The improvements in safety, efficiency and productivity will continue to increase as drone technology improves by enabling longer flights and concomitant scanning times. The technology will reduce in size allowing access into tighter spaces and becoming more robust to allow operation in even more difficult environments. Advances in sensor technology will mean more accurate and precise results, with longer ranges and a higher resolution, while the streamlining of data analytics will get results into the hands of the engineers faster, speeding up the decision-making processes. In the end, these outcomes will assist mining companies to achieve their aim of removing personnel from hazardous underground environments, while improving knowledge of the rock mass in every mining discipline.

The use of this technology is not limited to underground mining environments. Surface applications for LiDAR and drones has also seen an increased uptake in recent years.

\section{ACKNOWLEDGEMENTS}

Emesent is grateful to Barrick and Northern Star Resources for their continual assistance during the site trials period.

\section{REFERENCES}

Brady, B., Brown, E. (2004). Rock Mechanics for Underground Mining (3rd Edition), Dordrecht, The Netherlands, Kluwer Academic Publishers.

Jones, E., Beck, D. (2017), The use of three-dimensional laser scanning for deformation monitoring in underground mines, Proceedings of the 13th AusIMM Underground Operators Conference, AusIMM, Gold Coast, Australia.

Jones, E., Ghabraie, B., Beck, D. (2018), A method for determining the field accuracy of mobile laser scanning devices for Geomechanics, Proceedings of the Asian Rock Mechanics Symposium 10, Singapore, Singapore.

Zlot, R. and Bosse, M. (2012). Efficient Large-Scale 3D Mobile Mapping and Surface Reconstruction of an Underground Mine. Journal of Field Robotics. 31 (5): 758-779. 


\section{Evan Jones}

\section{Emesent}

Senior Mining and Geotechical Engineer

Evan is a senior mining and geotechnical engineer at Emesent. He is currently completing his $\mathrm{PhD}$ through the Western Australian School of Mines on the use of mobile scanning methods for the recognition of large-scale instability in underground hard rock mines. He worked as an engineer on mines in Australia and consulted to sites in Australia, Canada, South Africa, Canada, Indonesia, Chile, India and Spain. 